\title{
CLASSIFICATION OF LOCALLY EUCLIDEAN SPACES
}

\author{
LEO SARIO
}

1. The classification of Riemann surfaces has largely reached its completion. The purpose of the present paper is to lay the foundation for a new intriguing field in the classification theory: Riemannian spaces with Euclidean metrics. The paper is self-contained, both for the Riemann surface expert and the reader whose main interest is with higher dimensions.

The significance of locally Euclidean spaces lies, first of all, in that their function-theoretic nature differs for dimensions $n>2$ and $n=2$. The existence or nonexistence of Green's functions and positive or bounded harmonic functions in $R^{n}$, punctured $R^{n}$, and in the punctured flat torus offer simple examples. A striking phenomenon is that, despite such differences, the basic inclusion relations remain valid. Moreover, capacities and null-classes can be defined for components of point sets in $R^{\prime}$.

These results are established by an extension of the linear operator method ([6], [7]). The main points of the generalized method are given in Nos. 2, 3, $8,17,21$, and 23-27. The significance of this extension is in the fact that the absence of such devices as conformal mappings, conjugate harmonic functions, the reflection principle, and doubling of bordered regions necessitates new tools.

Another promising aspect of higher dimensions is the introduction of new function classes (Nos. 29-34). In No.35 we give a list of questions, an essential part of our paper. The important unsolved problem on the strictness of the inclusion $O_{H B} \subset O_{H D}$, well-known for Riemann surfaces (No. 24), is typical of these.

Several interesting topics are meaningful only in locally Euclidean spaces. However, at the cost of somewhat heavier equipment, some of our reasoning can be generalized to arbitrary Riemannian spaces.

Received March 8, 1964.

The work was sponsored by the U. S. Army Research Office (Durham), Grant DA$\mathrm{ARO}$ (D)-31-124-G 4.99, University of California, Los Angeles. The author is also grateful to his former students Professors A. Gray, K. V. R. Rao, B. Rodin, and G. Weill, for their valued comments and suggestions. 


\section{$\S 1$. Two lemmas on harmonic functions}

2. We start with two simple properties of harmonic functions.

Let $E$ be a compact set in a locally Euclidean space $V$.

LemMa. Consider the family of harmonic functions $u$ in $V$ with sgn $u \mid E \neq$ const. There exists a constant $0<q<1$, independent of $u$, such that

$$
q \inf _{V} u \leqq u \mid E \leqq q \sup _{V} u \text {. }
$$

We shall actually prove slightly more: if $\min _{E} u \leqq 0$ and $\sup _{v} u \geqq 0$, then there is a $q \in(0,1)$ such that $u \mid E \leqq q \sup _{v} u$. The first inequality (1) then. follows on applying this result to $-u$.

Proof. If $\sup _{v} u=0$ or $\infty$, there is nothing to prove. In other cases we multiply by a constant so as to make $\sup _{v} u=1$. The functions $v=1-u$ then have the properties $\inf _{v} v=0$ and $\max _{r} v \geqq 1$. We are to find a constant $q_{1}>0$ such that $\min _{F} v \geqq q_{1}$.

Cover $E$ by a finite number of solid spheres $C_{m}, m=1, \ldots, N, \bar{C}_{m} \subset V$, with radii $r_{m}$ such that slightly smaller solid spheres $C_{m}^{\prime}$ concentric with $C_{m}$ and with radii $r_{m}^{\prime}=k r_{m}$ already cover $E$. We shall denote by $|z|$ the length of the vector $z=\left(x_{1}, \ldots, x_{n}\right) \in R^{n}$. The area of the unit hypersphere $|z|=1$ is

$$
\omega_{n}=\frac{2(\sqrt{\pi})^{n}}{\Gamma\left(\frac{n}{2}\right)}
$$

and the Poisson formula for $v(z), z \in C_{m}$, reads

$$
v(z)=\frac{r_{m}^{n-2}\left(r_{m}^{2}-|z|^{2}\right)}{\omega_{n}} \int \frac{v d \omega_{n}}{\left(|z|^{2}+r_{m}^{2}-2|z| r_{m} \cos \theta\right)^{n / 2}}
$$

where $\theta$ is the angle between the radius to $z$ and that to the integration point. For $z \in C_{m}^{\prime}$, (2) gives the Harnack inequality

$$
\frac{1-k}{(1+k)^{n-1}} v\left(z_{m}\right) \leqq v(z) \leqq \frac{1+k}{(1-k)^{n-1}} v\left(z_{m}\right),
$$

where $z_{m}$ is the center of $C_{m}$. For any two points $z, z^{\prime} \in C_{m}^{\prime}$ we have

$$
c \leqq \frac{v(z)}{v\left(z^{\prime}\right)} \leqq c^{-1} \text { with } c=\left(\frac{1-k}{1+k}\right)^{n} .
$$

We may suppose that $E$ is connected, for if this is not the case we first 
replace $E$ by a larger connected compact set in $V$ and cover it by spheres as above. By assumption there is a $z_{0} \in E$ with $v\left(z_{0}\right) \geqq 1$. This point can be connected with any point $z \in E$ by a sequence of points $z_{j} \in E, j=1, \ldots, j_{z} \leqq N$, $z_{j_{z}}=z$, such that any pair $z_{j-1}, z_{j}$ is in some $C_{m}^{\prime}$. We have found a constant $q_{1}=c^{N}>0$ with the desired property $v(z) \geqq q_{1}$ for all $z \in E$ and all $v$.

3. Given a locally Euclidean space $V$ and a point $z_{0} \in V$ consider regions $\Omega \subset \Omega^{\prime}$ of $V$ containing $z_{0}$. Let $u_{\Omega}$ be a uniquely determined harmonic function on $\Omega$.

Lemмa. If the Dirichlet integral $D_{\Omega}$ over $\Omega$ has the directed limit

$$
\lim _{\Omega \rightarrow V} D_{\Omega}\left(\boldsymbol{u}_{\Omega}-u_{\Omega^{\prime}}\right)=0,
$$

then $u_{\Omega}(z)-u_{\Omega}\left(z_{0}\right)$ converges uniformly in compact subsets to a harmonic limit

$$
v(z)=\lim _{\Omega \rightarrow V}\left(u_{\Omega}(z)-u_{\Omega}\left(z_{0}\right)\right) .
$$

Proof. For any $i=1, \ldots, n$, the partial derivative $u_{x_{i}}$ of a harmonic function $u$ is harmonic. Its value at the center $z$ of a solid sphere $C$ of radius $\delta$ and with volume $V_{\delta}$ is

$$
u_{x_{i}}(z)=\frac{1}{V_{\delta}} \int_{c} u_{x_{i}} d V
$$

where $d V$ is the volume element. On applying the Schwarz inequality and summing from $i=1$ to $i=n$ one obtains

$$
|\operatorname{grad} u|^{2} \leqq \frac{1}{V_{\delta}} \int_{C}|\operatorname{grad} u|^{2} d V=\frac{1}{V_{\delta}^{-}} D_{C}(u) .
$$

Given a compact set $E \subset V$ cover it with solid spheres $C_{m} \subset V, m=1, \ldots$, $N$, of radii $r_{m}$ such that the spheres $C_{m}^{\prime}$ concentric with the $C_{m}$ and of radii $r_{m}^{\prime}=r_{m}-\delta_{m}$ already cover $E$. Again we may assume that $E$ is connected and we join $z_{0}$ to any $z \in E$ by a sequence $z_{j}, j=1, \ldots, j_{z} \leqq N$, with $z_{j-1}$, $z_{j}$ in some $C_{m}^{\prime}$. The line segment $d_{j}$ from $z_{j-1}$ to $z_{j}$ has length $<2 r_{0}$, where $r_{0}=\max r_{m}$, and we find for $\delta=\min \delta_{m}$ and for harmonic $u$ in $G=\cup C_{m}$ that

$$
\left|u\left(z_{j}\right)-u\left(z_{j-1}\right)\right| \leqq 2 r_{0} \max _{d_{j}}|\operatorname{grad} u| \leqq 2 r_{0} V_{\delta}^{-\frac{1}{2}} \sqrt{D_{G}(\dot{u})}
$$

This implies

$$
\left|u(z)-u\left(z_{0}\right)\right| \leqq 2 r_{0} N V_{\delta}^{-1}{ }^{-1} \sqrt{D_{G}(u)}
$$


An application to $u(z)=u_{\Omega}(z)-u_{\Omega^{\prime}}(z)$ with $G \subset \Omega$ gives the desired result.

\section{$\S 2$. Normal operators and principal functions}

4. Let $\Omega$ be a region of a locally Euclidean space $V$. Designate by $C$ the solid unit sphere $|z|<1$ and by $P$ a coordinate hyperplane, $x_{1}=0$, say. We shall call $\Omega$ a bordered region of $V$ if

(a) $\partial \Omega$ is compact in $V$,

(b) every $z \in \partial \Omega$ has a neighborhood $N_{z}$ and a diffeomorphism $h$ of $N_{z}$ with $C$ such that $h\left(N_{z} \cap \partial \Omega\right)=C \cap P$ and $h\left(N_{z} \cap \Omega\right)$ is one of the two half-balls of $C-P$.

A bordered region $\Omega \subset V$ shall be called a regular region if

(c) $\Omega$ is compact in $V$,

(d) $\Omega$ and $V-\bar{\Omega}$ have the same boundary in $V$,

(e) each component of $V-\Omega$ is noncompact in $V$.

We note that the border of a bordered region has a well-defined continuously turning normal and we can speak of the flux

$$
\int_{\partial \Omega} \frac{\partial u}{\partial n} d S
$$

across $\partial \Omega$ of a sufficiently regular function $u$ in $\bar{\Omega}$. Here $d S$ is the area element of $\partial \Omega$ and $\frac{\partial}{\partial n}$ is the normal derivative exterior (or interior, if so specified) to $\Omega$.

5. A function is, by definition, harmonic in a set $E \subset V$ if it has a harmonic extension to an open set containing $E$. Let $f$ be harmonic on the border $\alpha=\partial \Omega$ of a bordered region $\Omega \subset V$. Suppose there is a function $u \in C^{1}$ in $\bar{\Omega}$ with $u \mid \alpha$ $=f, u \in H$ in $\Omega, u=L f$ in $\bar{\Omega}$, where $H$ denotes the space of harmonic functions and $L$ is an operator which satisfies the following conditions:

$$
\begin{gathered}
L f \mid \alpha=f, \\
L\left(c_{1} f_{1}+c_{2} f_{2}\right)=c_{1} L f_{1}+c_{2} L f_{2}, \\
\min f \leqq L f \leqq \max f, \\
\int_{\alpha} \frac{\partial L f}{\partial n} d S=0 .
\end{gathered}
$$

An operator with properties (6)-(9) will be called a normal operator.

For a regular $\Omega$ the operator solving the Dirichlet problem is trivially 
normal. In $\S 5$ we shall see that even in the general case of a bordered region there are normal operators. Their effect is that, in an intuitive sense, there is no source or sink of $L f$ on the "ideal boundary" $\beta$ of the region, i.e., $\beta$ is "removable" for $L f$.

Special siginificance to normal operators is given by the fact that on a noncompact bordered region there generally are several operators, each with its own extremal property.

6. Let $V$ be a locally Euclidean space, and $\bar{V}_{1}$ the complement of a regular subregion with border $\alpha_{1}$. On $\bar{V}_{1}$ let there be given a continuous function $\sigma$, harmonic in $V_{1}$, and a normal operator $L$. We are interested in the problem of constructing on $V$ a harmonic function $p$, to be called the principal function, that imitates the behavior of $\sigma$ in $V_{1}$. More precisely, we require that $p \mid V_{1}=\sigma$ $+L(p-\sigma)$. This means that, in the sense of No. $5, p-\sigma$ must have no singularity on the ideal boundary of $V$. We also set out to find explicit upper and lower bounds for $p-\sigma$ in terms of $\sigma$.

Suppose $V$ is given by removing a finite number of points $z_{j}, j=1, \ldots$, $N$, from a locally Euclidean space $V^{*}$. Then $V_{1}$ may consist of disjoint solid $n$-spheres $C_{j}$ punctured at their centers $z_{j}$, and of the complement $V_{1}^{*}=V^{*}-\Omega^{*}$ of a regular region $\Omega^{*} \subset V^{*}, \cup \bar{C}_{j} \subset \Omega^{*}$. In $C_{j}-z_{j}$ the function $\sigma$ can be a singularity function, e.g., $r^{2-n}$ or any of its partial derivatives of any order. In $V_{1}^{*}, \sigma$ can be an arbitrarily behaving harmonic function. For $L$ we may take different normal operators in the various components of $V_{1}$. Thus our problem is to construct, on an arbitrary locally Euclidean space $V^{*}$, a harmonic function with given singularities at a finite number of preassigned points, and with a given behavior near the ideal boundary of $V^{*}$.

The theory of principal functions derives its significance from the triple generality in the choice of $V_{1}, \sigma$, and $L$.

\section{§. The main existence theorem}

7. To construct principal functions $p$ in a locally Euclidean space $V$ we may assume that

$$
\sigma \mid \alpha_{1}=0
$$

Indeed, if this condition is not met, we replace $\sigma$ by $\sigma_{0}=\sigma-L \sigma$. Then $p=\sigma_{0}+L p$ 
is the desired function in $V_{1}$.

It is in the nature of the problem that $\sigma$ have vanishing flux :

$$
\int_{\alpha_{1}} \frac{\partial \sigma}{\partial n} d S=0
$$

The flux of $p$ vanishes by Stokes' formula, and (11) follows from (9).

The solution of the problem will be uniquely determined up to an additive constant. Suppose indeed $p^{\prime}, p^{\prime \prime}$ were two solutions. Then by the maximum principle,

$$
\max _{V-V_{1}}\left(p^{\prime}-p^{\prime \prime}\right)=\max _{\alpha_{1}}\left(p^{\prime}-p^{\prime \prime}\right)
$$

and by (8),

$$
\max _{\bar{V}_{1}}\left(p^{\prime}-p^{\prime \prime}\right)=\max _{\alpha_{1}}\left(p^{\prime}-p^{\prime \prime}\right)
$$

It follows that

$$
\max _{V}\left(p^{\prime}-p^{\prime \prime}\right)=\max _{\alpha_{1}}\left(p^{\prime}-p^{\prime \prime}\right),
$$

and one infers that $p^{\prime}-p^{\prime \prime}$ is constant on $V$.

We shall give an explicit expression for a solution $p$.

8. Let $V_{0}$ with border $\alpha_{0}$ be a regular region of $V$ such that $\alpha_{0} \subset V_{1}$ and $\alpha_{1} \subset V_{0}$. Our problem is to find $p \mid \alpha_{0}$. In fact, then $p$ is obtained on $V=V_{0} U$ $V_{1}$ from the identities

$$
p\left|V_{0}=L^{\prime} p, \quad p\right| V_{1}=\sigma+L p,
$$

where $L^{\prime}$ is the operator providing us with the solution of the Dirichlet problem in $V_{0}$. We set

$$
K=L L^{\prime}
$$

and obtain on $\alpha_{0}$

$$
p=\sigma+K p
$$

The $n$th iterate of $K$ will be denoted by $K^{n}$.

Let $q$ be the constant of Lemma 2 applied to the compact set $\alpha_{1}$ in the region $V_{0}$, and set

$$
\begin{gathered}
Q=\frac{1}{1-q}, \\
m=\min _{\alpha_{0}} \sigma, \quad M=\max _{\alpha_{0}} \sigma .
\end{gathered}
$$

We are ready to state the main existence theorem: 
THEOREM. Given a locally Euclidean space $V$, let $V_{1} \subset V$ be a boundary neighborhood with compact border $\alpha_{1}$, and $V_{0} \subset V$ a regular region with border $\alpha_{0} \subset V_{1}, \alpha_{1} \subset V_{0} . \quad O n V_{1}$ let $\sigma$ be a harmonic function satisfying conditions (10), (11), and let $L$ be a normal operator defined by (6)-(9). Then the function

$$
p=\sum_{0}^{\infty} K^{n} \sigma
$$

on $V_{1}$ gives by (12) the principal function $p$ on $V$ :

$$
p-\sigma=L p \text {. }
$$

The function satifies the inequalities

$$
\begin{gathered}
m Q \leqq p \mid V_{0} \leqq M Q \\
m Q \leqq p-\sigma \leqq M Q
\end{gathered}
$$

9. Proof. We are to show that $p=\sum_{0}^{\infty} K^{n} \sigma \mid \alpha_{0}$ converges uniformly. Then $K$ can be applied term by term, for

$$
\left|K \sum_{0}^{\infty} K^{n} \sigma-\sum_{1}^{m} K^{n} \sigma\right|=\left|K \sum_{m+1}^{\infty} K^{n} \sigma\right| \leqq\left|\sum_{m+1}^{\infty} K^{n} \sigma\right| \boldsymbol{\alpha}_{0} \mid,
$$

which tends to 0 as $m \rightarrow \infty$. We have $K p=\sum_{1}^{\infty} K_{\sigma}^{n}=p-\sigma$ as required by (14). The proof will be based on Lemma 2 .

Let $h$ be continuous in $\bar{V}_{0} \cap \bar{V}_{1}$ and harmonic in $V_{0} \cap V_{1}$ with $h\left|\alpha_{1}=0, h\right| \alpha_{0}$ $=$ const. such that $\int_{a_{1}}(\partial h / \partial n) d S=1$, the derivative here and later being interior to $V_{0} \cap V_{1}$ on $\alpha_{1}$, exterior to it on $\alpha_{0}$. By Green's formula we have for any $u \in C^{1}$ in $\bar{V}_{0} \cap \bar{V}_{1}$, harmonic in $V_{0} \cap V_{1}$, with $\int_{\alpha_{0}} \frac{\partial u}{\partial n} d S=0$,

$$
\int_{\alpha_{0}} u \frac{\partial h}{\partial n} d S=\int_{\alpha_{1}} u \frac{\partial h}{\partial n} d S
$$

This holds, in particular, for functions $u=\sigma, L^{\prime} \varphi, L \psi, K \varphi$ with any harmonic $\hat{\varphi}$, $\psi$ on $\alpha_{0}, \alpha_{1}$.

We claim that

$$
\int_{\alpha_{1}} K^{n} \sigma \frac{\partial h}{\partial n} d S=0
$$

For $n=0$ this is so by (10). Suppose this holds for $n=i$. Then the same integral over $\alpha_{0}$ vanishes, hence

$$
\int_{\alpha_{0}} L^{\prime} K^{i} \sigma \frac{\partial h}{\partial n} d S=0
$$


Here $\alpha_{0}$ can be replaced by $\alpha_{1}$, and then $L^{\prime}$ by $L L^{\prime}$. Equality (21) follows for $i+1$, and consequently for $n=0,1, \ldots$

Since $\partial h / \partial n \geqq 0$ on $\alpha_{1}$ we conclude that $\operatorname{sgn} K^{n} \sigma \mid \alpha_{1} \neq$ const. Lemma 2 and relation (8) give for $n=1$,

$$
q m \leqq K \sigma \mid \alpha_{0} \leqq q M .
$$

Each increment of $n$ brings another factor $g$ and we obtain

$$
q^{n} m \leqq K^{n} \sigma \mid \alpha_{0} \leqq q^{n} M
$$

We have shown that $\sum_{0}^{\infty} K^{n}{ }_{\sigma} \mid \alpha_{0}$ converges uniformly:

$$
Q m \leqq p \mid \alpha_{0} \leqq Q M .
$$

By the maximum principle the same bounds hold for $p \mid V_{0}$, hence for $p \mid \alpha_{1}$ and $p-\sigma \mid \alpha_{1}$ and consequently for $p-\sigma$ in $V_{1}$. This completes the proof of Theorem 8.

Our next task is to show the existence of operators $L$. We shall first consider regular regions, then noncompact bordered regions.

\section{$\S$ 4. Normal operators for regular regions}

10. Let $\Omega$ be a regular region with disconnected border of a locally Euclidean space $V$. Partition the components of the border into two disjoint sets $\alpha$ and $\beta$. Let $f \in H$ on $\alpha$ and consider the family $U$ of functions $u$ such that

$$
u \in C^{1} \text { in } \Omega, \quad u|\Omega \in H, \quad u| \alpha=f .
$$

There exists a function $\boldsymbol{u}_{0} \in U$ determined by the additional property $\partial \boldsymbol{u}_{0} / \partial n=0$ on $\beta$ (for existence see Fichera [3], p. 196) ${ }^{* 1}$. We set $u_{0}=L_{0} f$.

Define the function $u_{1} \in U$ by the conditions

$$
u_{1} \mid \beta=c(\text { const. }), \quad \int_{\alpha} \frac{\partial u_{1}}{\partial n} d S=0
$$

The existence of the constant $c$ is seen as follows. For $u \in U$ with $u \mid \beta=c$ the flux across $\alpha$ toward $\Omega$ increases with $c$. In fact, for $c^{\prime}<c^{\prime \prime}$ and the corresponding functions $u^{\prime}, u^{\prime \prime}$, the difference $v=u^{\prime \prime}-u^{\prime}$ satisfies $v|\alpha=0, v| \beta \geqq 0$, $v \mid \Omega \geqq 0, \partial v / \partial n \geqq 0$ on $\alpha$, hence $\int_{\alpha}(\partial v / \partial n) d S \geqq 0$. A similar reasoning shows

*) The author is indebted to Professor G. Weill for pointing out this reference. 
that the flux of $u_{m} \in U$ with $u_{m} \mid \beta=\min f$ is nonpositive and that of $u_{M} \in U$ with $u_{\Perp} \mid \beta=\max f$ is nonnegative. Consequently there is a $c \in(\min f, \max f)$ that gives vanishing flux. We set $\boldsymbol{u}_{1}=L_{1} f$.

11. We shall also introduce an operator $(P) L_{1}$ as follows. Take a partition $P$ of the components $\gamma_{k}, k=1, \ldots, k_{\Omega}$ of $\beta$ into disjoint sets $\beta_{j}, j=1, \ldots j_{\Omega}$.

LEMMA. There exists a function $(P) u_{1} \in U$ with

$$
\text { (P) } u_{1} \mid \beta_{j}=c_{j}(\text { const. }), \quad \int_{\beta_{j}} \frac{\partial(P) u_{1}}{\partial n} d S=0 .
$$

Proof. Choose disjoint regular regions $D_{\alpha}, D_{k} \subset \Omega, k=1, \ldots, k_{\Omega}$, with disjoint borders $\alpha \cup \alpha^{\prime}, \gamma_{k} \cup \gamma_{k}^{\prime}$ respectively. In $\bar{D}_{\alpha}$ take the function $u_{\alpha} \in C^{1}$, $u_{\alpha}\left|D_{\alpha} \in H, u_{\alpha}\right| \alpha=f, u_{\alpha} \mid \alpha^{\prime}=c_{\alpha}, \int_{\alpha}\left(\partial u_{\alpha} / \partial n\right) d S=0$. Apply Theorem 8 to $\Omega$ with $V_{1}=D_{\alpha} \cup D_{1} \cup \cdots \cup D_{k_{\Omega}}$ and $\sigma=u_{\alpha}-c_{\alpha}$ in $D_{\alpha}, \sigma=0$ in each $D_{k}$. For $L$ take in $D_{\alpha}$ and each $\bigcup_{\gamma_{k}<\beta_{j}} D_{k}$ the operator $L_{1}$. The resulting principal function $p$ is $f+c$ on $\alpha$ and the desired function is $(P) \boldsymbol{u}_{1}=p-c$. Write

$$
\text { (P) } u_{1}=(P) L_{1} f \text {. }
$$

12. The most important partitions are the identity partition where $j_{\Omega}=1$, and the canonical partition, where $j_{\Omega}=k_{Q}$. In the former case, $(P) L_{1}=L_{1}$. For the sake of simplicity we shall henceforth assume that a partition $P$ has been given in advance and we let $L_{1}$ and $u_{1}$ stand for $(P) L_{1}$ and $(P) u_{1}$.

With this understanding we take a real constant $\lambda$ and introduce

$$
\boldsymbol{u}_{\lambda}=(1-\lambda) \boldsymbol{u}_{0}+\lambda \boldsymbol{u}_{1}=L_{\lambda} f .
$$

Clearly $L_{\lambda}$ satisfies the conditions (6)-(9) of a normal operator, for so do $L_{0}$ and $L_{1}$.

13. Let $U_{0} \subset U$ be the class of functions $u$ with the additional property

$$
\int_{p_{j}} \frac{\partial u}{\partial n} d S=0, j=1, \ldots, j_{\Omega}
$$

For $u, v \in U_{0}$ we set

$$
\begin{aligned}
& A(u)=\int_{\alpha} u \frac{\partial u}{\partial n} d S, \quad A(u, v)=\int_{\alpha} u \frac{\partial v}{\partial n} d S, \\
& B(u)=\int_{\beta} u \frac{\partial u}{\partial n} d S, \quad B(u, v)=\int_{\beta} u \frac{\partial v}{\partial n} d S .
\end{aligned}
$$


The Dirichlet integral of $u$ over $\Omega$ will be denoted by $D(u)$.

LeMMA. The function $u_{\lambda}$ minimizes the functional $B(u)+(2 \lambda-1) A(u)$ in $U_{0}$. Explicitly,

$$
B(u)+(2 \lambda-1) A(u)=\lambda^{2} A\left(u_{1}\right)-(1-\lambda)^{2} A\left(u_{0}\right)+D\left(u-u_{\lambda}\right) .
$$

Thus the value of the minimum is $\lambda^{2} A\left(u_{1}\right)-(1-\lambda)^{2} A\left(u_{0}\right)$ and the deviation from this minimum is $D\left(u-u_{\lambda}\right)$.

Proof. In view of $\boldsymbol{u}-\boldsymbol{u}_{\lambda} \mid \alpha=0$ we have

$$
D\left(u-u_{\lambda}\right)=B(u)+B\left(u_{\lambda}\right)-B\left(u, u_{\lambda}\right)-B\left(u_{\lambda}, u\right) .
$$

Here

$$
\begin{aligned}
B\left(u_{\lambda}\right) & =\lambda(1-\lambda) B\left(u_{0}, u_{1}\right) \\
& =\lambda(1-\lambda)\left(B\left(u_{0}, u_{1}\right)-B\left(u_{1}, u_{0}\right)\right) \\
& =\lambda(1-\lambda)\left(A\left(u_{1}\right)-A\left(u_{0}\right)\right) .
\end{aligned}
$$

Similarly,

$$
\begin{aligned}
B\left(\boldsymbol{u}, u_{\lambda}\right) & =\lambda\left(B\left(\boldsymbol{u}, u_{1}\right)-B\left(u_{1}, u\right)\right) \\
& =\lambda\left(A\left(u_{1}\right)-A(u)\right)
\end{aligned}
$$

and

$$
\begin{aligned}
B\left(u_{\lambda}, u\right) & =(1-\lambda)\left(B\left(u_{0}, u\right)-B\left(u, u_{0}\right)\right) \\
& =(1-\lambda)\left(A(u)-A\left(u_{0}\right)\right) .
\end{aligned}
$$

Equation (30) follows.

\section{$\S 5$. Normal operators for noncompact regions}

14. Let $V_{1}$ be a noncompact bordered region, with border $\alpha$, of a locally Euclidean space $V$. Take a regular region $\Omega \subset V_{1}$ with border $\alpha \cup \beta_{\Omega}$. We shall consider partitions $\beta_{\Omega j}$ of $\beta_{\Omega}$ such that the border of any component of $V_{1}-\Omega$ belongs to exactly one $\beta_{\Omega j}$. A partition $\left\{\beta_{\Omega^{*}}^{*}\right\}$ of $\beta_{\Omega}, j^{*}=1, \ldots, j_{\Omega}^{*}$, is said to be a refinement of a partition $\left\{\beta_{\Omega j}\right\}, j=1, \ldots, j_{\Omega}$, if $\beta_{\Omega j^{*}}^{*}$ is contained in only one $\beta_{\Omega j}$.

Let $G_{\Omega j}$ be the union of those components of $V_{1}-\Omega$ whose border belongs to $\beta_{\Omega j}$. Let $\Omega \subset \Omega^{\prime}, \partial \Omega^{\prime}=\alpha \cup \beta_{\Omega^{\prime}}, \beta_{\Omega} \subset \Omega^{\prime}$. A partition $\left\{\beta_{\Omega^{\prime} j}\right\}$ of $\beta_{\Omega^{\prime}}$ is said to be induced by the partition $\left\{\beta_{\Omega j}\right\}$ of $\beta_{\Omega}$ if $\beta_{\Omega^{\prime} j}=\beta_{\Omega^{\prime}} \cap G_{\Omega j}$. Partitions of the $\beta_{\Omega}$ for all $\Omega$ are said to form a consistent system of partitions if for $\Omega \subset \Omega^{\prime}$ the partition of $\beta_{\Omega}$, is a refinement of the partition induced by that of $\beta_{\Omega}$. We shall only 
consider consistent systems of partitions. The most important systems are the identity partition, where $\beta_{\Omega}$ constitutes one part $\beta_{\Omega j}$ only, and the canonical partition, where $\beta_{\Omega j}$ is the border of exactly one component of $V_{1}-\Omega$.

15. Given a consistent system of partitions, the function $\boldsymbol{u}_{\Omega \lambda}$ and the operator $L_{\Omega \lambda}$ are formed in each regular $\Omega$ as in Nos. 10-12. Note that we cannot prove the existence of the directed limit of $u_{\Omega \lambda}$ by Lemma 3 . The reason is that the points $z_{0}$ where we know that the limit exists are on the border $\alpha$, not interior to $V_{1}$. In the 2-dimensional case the difficulty can be overcome by choosing $\alpha$ to be an analytic Jordan curve and by forming the double $\hat{V}_{1}$ of $V_{1}$. But for $n>2$ such reflection is not possible. For this reason we shall, in this section, make use of normal families.

The functions $u_{\Omega \lambda}$ are uniformly bounded between $\min f$ and $\max f$. Every nested sequence $\left\{\Omega_{m}\right\}$ with $\Omega_{m} \rightarrow V_{1}$ as $m \rightarrow \infty$ has a subsequence, again denoted by $\left\{\Omega_{m}\right\}$, for which the corresponding functions $u_{m \lambda}$ converge uniformly in compact subsets of $V_{1}$. By the maximum principle the convergence is uniform in $\bar{V}_{1}$, and the limiting function $\boldsymbol{u}_{\lambda}$ is continuous on $\bar{V}_{1}$, harmonic on $V_{1}$.

Every limiting function $u_{\lambda}$ belongs to the class $U_{0}$ of functions $u \in C^{1}$ in $\tilde{V}_{1}$, $u\left|V_{1} \in H, u\right| \alpha=f$, and

$$
\int_{\beta \Omega 2 j} \frac{\partial u}{\partial n} d S=0
$$

for every $\beta_{\Omega j}$ in the given consistent system of partitions.

16. Let

$$
B_{\Omega}(u)=\int_{\beta_{\Omega}} u \frac{\partial u}{\partial n} d S, \quad B_{\Omega}(u, v)=\int_{\beta_{S}} u \frac{\partial v}{\partial n} d S,
$$

and define

$$
B(u)=\lim _{\Omega \rightarrow V_{1}} \int_{\beta_{\Omega}} u \frac{\partial u}{\partial n} d S,
$$

where symbolically $B(u)$ is the integral over the ideal boundary of $V_{1}$.

By way of preparation of (30) for the noncompact $V_{1}$ we first prove:

Lemma. Any limiting function $u_{\lambda}=\lim _{m \rightarrow \infty} u_{m \lambda}$ minimizes the functional $B(u)+(2 \lambda-1) A(u)$ in $U_{0}$.

Proof. Let $u_{m}=u_{m \lambda}$ and

$$
F(u)=B(u)+(2 \lambda-1) A(u),
$$




$$
F_{m}(u)=B_{m}(u)+(2 \lambda-1) A(u)
$$

where $B_{m}$ refers to $\beta_{m} \subset \partial \Omega_{m}$. Then

$$
F\left(u_{\lambda}\right)=\lim _{m \rightarrow \infty} F_{m}\left(u_{\lambda}\right)=\lim _{m \rightarrow \infty} \lim _{n \rightarrow \infty} F_{m}\left(u_{n}\right) . \quad \text { For } m \leqq n . F_{m}\left(u_{n}\right) \leqq F_{n}\left(u_{n}\right)
$$

and consequently

$$
F\left(\boldsymbol{u}_{\lambda}\right) \leqq \lim \inf _{n \rightarrow \infty} F_{n}\left(\boldsymbol{u}_{n}\right)
$$

On the other hand,

$$
F_{n}\left(u_{n}\right) \leqq F_{n}(u) \leqq F(u)
$$

for all $u \in U_{0}$. It follows that

$$
\lim \sup _{n \rightarrow \infty} F_{n}\left(u_{n}\right) \leqq \inf _{U_{0}} F(u) \leqq F\left(u_{\lambda}\right) .
$$

We conclude from (34) and (35) that

$$
\min _{U_{0}} F(u)=F\left(u_{\lambda}\right)=\lim _{n \rightarrow \infty} F_{n}\left(u_{n}\right) .
$$

17. We are ready to state:

THEOREM. On an arbitrary bordered region $V_{1}$, compact or not, of a locally Euclidean space there is a unique function $u_{\lambda}$ which in $U_{0}$ has the extremal property

$$
B(u)+(2 \lambda-1) A(u)=\lambda^{2} A\left(u_{1}\right)-(1-\lambda)^{2} A\left(u_{0}\right)+D\left(u-u_{\lambda}\right) .
$$

Proof. For any limiting function $\boldsymbol{u}_{\lambda}=\lim \boldsymbol{u}_{n}$ and for $u \in U_{9}$ set $\boldsymbol{u}-\boldsymbol{u}_{\lambda}=\boldsymbol{h}$. Then $u_{\lambda}+\varepsilon h \in U_{0}$ for any real $\varepsilon$ and

$$
\begin{array}{r}
F_{n}\left(u_{\lambda}+\varepsilon h\right)=F_{n}\left(u_{\lambda}\right)+\varepsilon^{2} D_{n}(h) \\
+\varepsilon\left[B_{n}\left(u_{\lambda}, h\right)+B_{n}\left(h, u_{\lambda}\right)\right. \\
\left.+(2 \lambda-1) A\left(u_{\lambda}, h\right)\right] .
\end{array}
$$

Suppose $D(h)<\infty$. As $n \rightarrow \infty$ the first three terms have limits and, as a consequence, the bracketed expression $I_{n} \rightarrow I$ :

$$
F\left(\boldsymbol{u}_{\lambda}+\varepsilon h\right)=F\left(u_{\lambda}\right)+\varepsilon^{2} D(h)+\varepsilon I .
$$

By the minimum property of $u_{\lambda}$ we have $d F / d \varepsilon=0$ for $\varepsilon=0$, hence $I=0$. On setting $\varepsilon=1$ we obtain the desired deviation formula

$$
F(u)=F\left(u_{\lambda}\right)+D\left(u-u_{\lambda}\right) .
$$

The formula remains valid in a degenerate form for $D(h)=\infty$. 
Suppose $u^{\prime}, u^{\prime \prime}$ are two minimizing functions. Then

$$
D\left(u^{\prime}-u^{\prime \prime}\right)=F\left(u^{\prime}\right)-F\left(u^{\prime \prime}\right)=0
$$

and the uniqueness follows.

Corollary. $u_{0}$ minimizes $D(u), u_{1}$ minimizes $A(u)+B(u)$ in $U_{0}$.

18. The uniqueness established, we can write

$$
u_{\lambda}=L_{\lambda} f
$$

where $L_{\lambda}$ is a normal operator satisfying conditions (6)-(9). Indeed, the approximating operators $L_{\Omega \lambda}$ were seen to enjoy these properties and the same is true of the limiting operators because of uniform convergence.

The principal functions $p_{\lambda}$ corresponding to the $L_{\lambda}$ possess important minimal properties which we proceed to establish.

\section{§ 6. Extremal properties of principal functions}

19. First let $\Omega$ be a regular region with border $\beta$. Take two solid spheres $C_{a}, C_{b}$ centered at $a, b$ and with disjoint closures $\bar{C}_{a}, \bar{C}_{b} \subset \Omega$. Consider the class $P$ of functions $p \in C^{1}$ in $\bar{\Omega}-a-b, p \mid \Omega-a-b \in H$ and with the following properties :

$$
\begin{gathered}
p \mid \bar{C}_{a}=\frac{|z-a|^{2-n}}{\omega_{n}(n-2)}+h(z), \\
p \mid \bar{C}_{b}=-\frac{|z-b|^{2-n}}{\omega_{n}(n-2)}+k(z), k(b)=0 \\
\int_{\beta j} \frac{\partial p}{\partial n} d S=0 .
\end{gathered}
$$

Here $\omega_{n}$ is the area $2 \pi^{n / 2} / \Gamma(n / 2)$ of the unit hypersphere $|z|=1 ; h, k$ are harmonic in $\bar{C}_{a}, \bar{C}_{b}$, and $\left\{\beta_{j}\right\}$ is a given partition of $\beta$. We let $h_{0}, h_{1}$ signify the $h$ corresponding to $p_{0}, p_{1}$.

LeMmA. On a regular region $\Omega$ of a locally Euclidean space the function $p_{\lambda}$ has the property

$$
B(p)+(2 \lambda-1) h(a)=\lambda^{2} h_{1}(a)-(1-\lambda)^{2} h_{0}(a)+D\left(p-p_{\lambda}\right) .
$$

Proof. For short we write $r$ for $|z-a|$ or $|z-b|$ and set

$$
s(r)=\frac{r^{2-n}}{\omega_{n}(n-2)} .
$$


The flux of $p$ across $\alpha_{a}=\partial C_{a}$ away from the center is -1 , and that across $\alpha_{b}=\partial C_{b}$ away from the center is +1 .

We again start with

$$
D\left(p-p_{\lambda}\right)=B(p)+B\left(p_{\lambda}\right)-B\left(p, p_{\lambda}\right)-B\left(p_{\lambda}, p\right)
$$

and use $A_{a}, A_{b}$ for $A$ taken over $\alpha_{a}, \alpha_{b}$. Here

$$
B\left(p_{\lambda}\right)=\lambda(1-\lambda)\left(B\left(p_{0}, p_{1}\right)-B\left(p_{1}, p_{0}\right)\right)
$$

is the sum of

$$
\lambda(1-\lambda)\left[A_{a}\left(s+h_{0}, s+h_{1}\right)-A_{a}\left(s+h_{1}, s+h_{0}\right)\right]
$$

and a similar expression containing $A_{b}$. In the bracketed quantity, $A_{a}\left(s, h_{i}\right)$ $=0$ for $i=0,1, A_{a}\left(h_{0}, h_{1}\right)-A_{a}\left(h_{1}, h_{0}\right)=0$, and the only nonvanishing terms are

$$
A_{a}\left(h_{0}, s\right)-A_{a}\left(h_{1}, s\right)=h_{1}(a)-h_{0}(a) .
$$

Because of the normalization $k(b)=0$ the corresponding expression for $A_{b}$ vanishes and we obtain

$$
B\left(p_{\lambda}\right)=\lambda(1-\lambda)\left(h_{1}(a)-h_{0}(a)\right) .
$$

Analogous computations yield

$$
\begin{aligned}
& B\left(p, p_{\lambda}\right)=\lambda\left(h_{1}(a)-h(a)\right), \\
& B\left(p_{\lambda}, p\right)=(1-\lambda)\left(h(a)-h_{0}(a)\right),
\end{aligned}
$$

and (41) follows.

20. If $V$ is a locally Euclidean space, let a consistent system of partitions be given for the borders $\beta_{\Omega}$ of all regular subregions $\Omega \subset V$ that contain $a$ and $b$. Let $\Omega \subset \Omega^{\prime}$ with $\beta_{\Omega} \subset \Omega^{\prime}$ and denote by $p_{\lambda}, h_{\lambda}, B$ quantities corresponding to $\Omega$, and by $p_{\lambda}^{\prime}, h_{\lambda}^{\prime}, B^{\prime}$ those corresponding to $\Omega^{\prime}$. For $p=p_{0}^{\prime} \mid \Omega, p_{\lambda}=p_{0}, \quad(41)$ gives

$$
B\left(p_{0}^{\prime}\right)-h_{0}^{\prime}(a)=-h_{0}(a)+D\left(p_{0}^{\prime}-p_{0}\right)
$$

for $p=p_{1}^{\prime} \mid \dot{\Omega}, p_{\lambda}=p_{1}$

$$
B\left(p_{1}^{\prime}\right)+h_{1}^{\prime}(a)=h_{1}(a)+D\left(p_{1}^{\prime}-p_{1}\right)
$$

for $p=p_{1}, p_{\lambda}=p_{0}$,

$$
B\left(p_{1}\right)-h_{1}(a)=-h_{0}(a)+D\left(p_{1}-p_{0}\right)
$$


By virtue of $B\left(p_{i}\right)=0, B\left(p_{i}^{\prime}\right) \leqq B^{\prime}\left(p_{i}^{\prime}\right)=0$, we have:

Lemma. $h_{0}(a)$ decreases, $h_{1}(a)$ increases with increasing $\Omega$ and $h_{1}(a) \leqq h_{0}(a)$ for every $\Omega$.

One concludes that the directed limits $h_{i}(a)=\lim _{\Omega \rightarrow 1} \cdot h_{i \Omega}(a), i=0,1$, exist and so do

$$
\lim _{\substack{\Omega \rightarrow \Gamma^{\prime} \\ \Omega \subset \Omega^{\prime}}} D_{\Omega}\left(p_{\Omega i}-p_{\Omega^{\prime} i}\right)=0 .
$$

From this and from the normalization $p_{\Omega}(b)-p_{\Omega^{\prime}}(b)=0$ Lemma 3 gives the harmonic directed limits

$$
p_{i}=\lim _{\Omega \rightarrow \Gamma} p_{\Omega i}
$$

on $V-a-b$, the convergence being uniform in compact subsets. Write

$$
p_{\lambda}=(1-\lambda) p_{0}+\lambda p_{1} .
$$

21. We consider the family $P$ of functions $p \in H$ on $V-a-b$ with singularities (38), (39) and the property

$$
\int_{R_{S 2 j}} \frac{\partial p}{\partial n} d S=0
$$

for all $\beta_{\Omega j}$ in the given partition.

To establish the extremal property (41) of $p_{\lambda}$ in $P$ for the noncompact $V$ we let $\Omega^{\prime} \rightarrow V$ in (42), (43) and obtain

$$
\begin{aligned}
& B_{\Omega}\left(p_{0}\right)-h_{0}(a)=-h_{\Omega 0}(a)+D_{\Omega}\left(p_{0}-p_{\Omega 0}\right), \\
& B_{\Omega}\left(p_{1}\right)+h_{1}(a)=h_{\Omega 1}(a)+D_{\Omega}\left(p_{1}-p_{\Omega 1}\right) .
\end{aligned}
$$

On letting $\Omega \rightarrow V$ we infer by $B_{\Omega} \leqq 0$ that

$$
\lim _{\Omega \rightarrow r} D_{\Omega 2}\left(p_{i}-p_{\Omega i}\right)=0
$$

for $i=0,1$.

By virtue of the triangle inequality this gives

$$
\lim _{\Omega \rightarrow r^{-}} D_{\Omega}\left(p_{\lambda}-p_{\Omega \lambda}\right)=0 .
$$

From this and the definition $D\left(p-p_{\lambda}\right)=\lim _{\Omega \rightarrow r} D_{\Omega \Omega}\left(p-p_{\lambda}\right)$ one concludes, again by the triangle inequality, that

$$
\lim _{\Omega \rightarrow V} D_{\Omega}\left(p-p_{\Omega \lambda}\right)=D\left(p-p_{\lambda}\right) .
$$

The deviation formula for $\Omega$ and $p \in P$ on $V$ reads 


$$
B_{\Omega}(p)+(2 \lambda-1) h(a)=\lambda^{2} h_{\Omega 1}(a)-(1-\lambda)^{2} h_{\Omega 0}(a)+D_{\Omega}\left(p-p_{\Omega \lambda}\right) .
$$

We let $\Omega \rightarrow V$ and obtain what we set out to find:

Theorem. In the class $P$ in a locally Euclidean space $V$ the function $p_{\lambda}$. minimizes the functional $F(p)=B(p)+(2 \lambda-1) h(a)$. The value of the minimum is $\lambda^{2} h_{1}(a)-(1-\lambda)^{2} h_{0}(a)$ and the deviation of $F(p)$ from this minimum is $D\left(p-p_{\lambda}\right)$.

It is an open question what is the extremal property of $p_{\lambda}$ if the singularities $s,-s$ of (38), (39) are replaced by partial derivatives of $s$.

For later reference we observe that

$$
B\left(p_{0}\right)=B\left(p_{1}\right)=0
$$

This follows by choosing $p=p_{i}, p_{\lambda}=p_{i}, i=0,1$.

22. We have these immediate consequences:

Corollary 1. The function $p_{0}$ gives to $B(p)-h(a)$ the minimum $-h_{0}(a)$, and the function $p_{1}$ gives to $B(p)+h(a)$ the minimum $h_{1}(a)$, both in $P$.

Corollary 2. Among functions in $P$ with $B(p) \leqq 0$, we have

$$
h_{1}(a) \leqq h(a) \leqq h_{0}(a) \text {. }
$$

Definition. The span of the region $V$ is

$$
S=h_{0}(a)-h_{1}(a) \text {. }
$$

Observe that the span depends on the class $P$, i.e., on $a, b$, and the system of partitions.

Corollary 3. The function $\frac{1}{2}\left(p_{0}+p_{1}\right)$ gives the minimum

$$
\min _{P} B(p)=-\frac{S}{4}
$$

In particular, $B(p) \geqq 0$ for all $p \in P$ if and only if the span vanishes. We shall return to the span in Nos. $23,24$.

The function $p_{0}-p_{1}$ is not in $P$, and a separate discussion will be needed to establish its extremal property.

\section{§ 7. Extremal harmonic functions}

23. Let $Q$ be a regular region of a locally Euclidean space. For any real 
$\mu$, $\lambda$ let

$$
p_{\mu \lambda}=\mu p_{0}+\lambda p_{1}
$$

where $p_{0}, p_{1}$ are in $P$, defined for the compact $\bar{\Omega}$ (No. 19). We introduce the class $Q$ of functions $q \in C^{1}$ in $\bar{\Omega}-a-b, q \in H$ in $\Omega-a-b$, and with the additional properties

$$
\begin{gathered}
q \mid \bar{C}_{a}=(\mu+\lambda) s+e, \\
q \mid \bar{C}_{b}=-(\eta+\lambda) s+f, \quad f(b)=0, \\
\int_{\beta j} \frac{\partial q}{\partial n} d S=0,
\end{gathered}
$$

$j=1, \ldots, j_{\Omega}$, where $e, f \in H$ in $\bar{C}_{a}, \bar{C}_{b}$. If $\mu+\lambda=0$, then $q \in C^{1}$ in $\bar{\Omega}, q \mid \Omega \in H$.

We retain the meaning of $h_{0}, h_{1}$ for $p_{0}, p_{1} \in P$ and state:

THEOREM. The function $p_{\mu \lambda}$ in $Q$ has the minimum property

$$
B(q)+(\lambda-\mu) e(a)=\lambda^{2} h_{1}(a)-\mu^{2} h_{0}(a)+D\left(q-p_{\mu \lambda}\right) .
$$

The proof is an analogue of that in No. 19 when we note that the singularity at $a$ of $q$ is $(\mu+\lambda) s$ while that of $p_{0}$ and $p_{1}$ is $s$. The intermediate results are now

$$
\begin{aligned}
& B\left(p_{\mu \lambda}\right)=\mu \lambda\left[h_{1}(a)-h_{0}(a)\right], \\
& B\left(q, p_{\mu \lambda}\right)=\lambda\left[(\mu+\lambda) h_{1}(a)-e(a)\right], \\
& B\left(p_{\mu \lambda}, q\right)=\mu\left[e(a)-(\mu+\lambda) h_{0}(a)\right],
\end{aligned}
$$

and (54) follows.

In the case of a noncompact locally Euclidean space $V$ the only change in defining the class $Q$ is that the flux of $q \in Q$ is to vanish across every $\beta_{\Omega j}$ in a given consistent system of partitions. Since the convergence proof of $p_{\Omega \mu \lambda}$ is based on that of $p_{\Omega 0}, p_{\Omega 1}$, the reasoning in 20-21 applies mutatis mutandis. We conclude that the deviation formula (54) holds for the limiting function $p_{\mu \lambda}$ in $V$.

The main motivation for considering $\mu, \lambda$ without the earlier restriction $\mu+\lambda=1$ is that we can now have $\mu+\lambda=0$. The competing class $Q$ then is the class $U$ of regular harmonic functions $u$ on $V$ with the normalization $u(b)$ $=0$ and with vanishing flux across each $\beta_{\Omega j}$.

THEOREM. The function $p_{0}-p_{1}$ has the following minimum property in $U$ :

$$
D(u)-2 u(a)=-S+D\left(u-p_{0}+p_{1}\right) .
$$


On setting $u=0$ we obtain:

COROLlARY. The span has the value

$$
S=D\left(p_{0}-p_{1}\right)
$$

and $p_{0}=p_{1}$ if and only if the span vanishes.

24. The class $H D$ for a given locally Euclidean space $V$ consists, by definition, of harmonic functions with a finite Dirichlet integral over $V$. A space $V$ is in $O_{H D}$ if there are no nonconstant $H D$-functions in $V$.

THEOREM. $V \notin O_{H D}$ if and only if $\mathrm{S} \neq 0$ for some $a, b$ and the identity partition.

Proof. From (46) and the triangle inequality we conclude that $p_{0}-p_{1} \in H D$. Suppose there is a nonconstant $u \in H D$ in $V$. Then there is a nonconstant $u \in H D$ in $V$ with $u(b)=0$. Let $a \in V$ be a point for which $u(a) \neq 0$. If $p_{0}-p_{1}$ were constant, we would conclude from

$$
D(u)-2 u(a)=-D\left(p_{0}-p_{1}\right)+D\left(u-p_{0}+p_{1}\right)
$$

that $u(a)=0$. Thus $S=D\left(p_{0}-p_{1}\right) \neq 0$.

Conversely, if $S \neq 0$, then $p_{0}-p_{1}$ is a nonconstant $H D$.function in $V$.

Let $H B$ be the class of harmonic bounded functions in $V$.

Lemma. The existence of non ronstant HD-functions in $V$ implies that of nonsonstant HB-functions:

$$
O_{H B} \subset O_{H D}
$$

In fact, $p_{0}-p_{1}=L\left(p_{0}-p_{1}\right)$ is bounded in a boundary neighborhood, hence in $V$.

Other $O$-classes of interest are introduced in $\S 9$.

\section{$\S 8$. Capacity functions}

25. We shall introduce the capacity of the ideal boundary and of a boundary component of a locally Euclidean space $V$.

Consider a regular region $\Omega \subset V$ with border $\beta=\gamma \cup \beta_{1} \cup \cdots \cup \beta_{j \varepsilon}$, where $\gamma$ is a set of components of $\beta$ and each $\beta_{j}, j=1, \ldots, j_{\Omega}$, is a component of $\beta-\gamma$.

Let $C_{a}$ be a solid $n$-sphere centered at a given point $a$, with $\bar{C}_{a} \subset \Omega$. Denote by $P$ the class of functions $p \subseteq C^{1}$ on $\Omega-a, p \in H$ in $\Omega-a$, 


$$
\begin{gathered}
p \mid \bar{C}_{a}=-\frac{|z-a|^{2-n}}{\omega_{n}(n-2)}+h(z), \\
\int_{r} \frac{\partial p}{\partial n} d S=1, \\
\int_{\beta j} \frac{\partial p}{\partial n} d S=0, \quad j=1, \ldots, j_{\Omega} .
\end{gathered}
$$

Here $h \in H$ and $h(a)=0$. Clearly (59) is a consequence of $(60)$.

In $P$ the capacity function $p_{r}$ of $r$ is defined by the properties

$$
\begin{aligned}
& p_{r} \mid \gamma=k_{r}, \\
& p_{r} \mid \beta_{j}=k_{j},
\end{aligned}
$$

$k_{r}, k_{j}$ being constants. The existence can easily be established by the main existence theorem (No. 8).

ThEOREM. The capacity function minimizes $B(p)$ in $P$ :

$$
\min _{P} B(p)=k_{r}+D\left(p-p_{r}\right) .
$$

Proof. On adding to the right side of

$$
D\left(p-p_{r}\right)=B\left(p, p-p_{r}\right)
$$

the quantity

$$
B\left(p_{\curlyvee}, p\right)-B\left(p_{\curlyvee}\right)=0
$$

one obtains

$$
D\left(p-p_{r}\right)=B(p)-B\left(p_{r}\right)+B\left(p_{r}, p\right)-B\left(p, p_{r}\right) .
$$

One transfers $B\left(p_{r}, p\right)-B\left(p, p_{r}\right)$ to $\partial C_{a}$ and shows in the same fashion as in No. 19 that its value is $h_{r}(a)-h(a)$, hence 0 . This proves the theorem.

26. In passing we note that for $\gamma=\beta, p_{3}$ also has the following extremal property.

THEOREM. The capacity function $p_{\beta}$ of the border $\beta$ of a regular region gives

$$
\min _{P} \sup _{\Omega} p=\sup _{\Omega} p_{\beta}=k_{\beta} \text {. }
$$

In fact, for any harmonic function $u$

$$
B\left(u, p_{3}\right)=u(a)
$$

In particular, this is true for $u=p-p_{\beta}$. Since $B\left(p_{\beta}\right)=k_{\beta}$, it follows from $u(a)$ $=0$ that $B\left(p, p_{i}\right)=k_{i}$, and the possibility of $\sup _{\Omega} p<k_{\beta}$ is excluded. 
27. Let $\left\{\Omega_{n}\right\}$ be a nested sequence of reguiar regions of a locally Euclidean space $V$ with $\cup \Omega_{n}=V$. Consider a consistent system $\left\{\beta_{n j}\right\}$ of partitions of the $\left\{\partial \Omega_{n}\right\}$. A sequence $\left\{\gamma_{n}\right\}=\left\{\beta_{n j(n)}\right\}$ defines a subboundary $\gamma$ of the ideal boundary $\beta$ of $V$ if $\beta_{n+1, j(n+1)}$ is in the component of $V-\Omega_{n}$ bordered by $\beta_{n j(n)}$. Equivalence in two exhaustions is defined in an obvious manner. For the identity partition $\gamma$ is the ideal boundary $\beta$. For the canonical partition each $\gamma$ is a boundary component. In general $\gamma$ is a boundary component if each $\gamma_{n}$ in the sequence defining $\gamma$ is the border of exactly one component of $V-\Omega_{n}$.

For a regular $\Omega \subset V$ let $\beta_{\Omega\}}$ be the part of $\beta_{\Omega}$ that corresponds to a given $\gamma$. Let $p_{\Omega \text { r }}$ be the capacity function of $\gamma_{\Omega}$ on $\bar{\Omega}$ with $\left.p_{\Omega \gamma}\right|_{\gamma_{\Omega}}=k_{\Omega \text { r }}$.

LemMA. For $\bar{\Omega} \subset \Omega^{\prime}$,

$$
k_{\Omega i} \leqq k_{\Omega^{\prime} \gamma}
$$

Indeed,

$$
k_{Q \gamma}=B_{\Omega}\left(p_{\Omega}\right) \leqq B_{\Omega}\left(p_{\Omega^{\prime} \gamma}\right) \leqq B_{\Omega^{\prime}}\left(p_{\Omega^{\prime} \gamma}\right)=k_{\Omega^{\prime} \gamma} .
$$

We conclude that the directed limit exists :

$$
k_{\Upsilon}=\lim _{\Omega \rightarrow V} k_{\Omega \Upsilon} .
$$

In the case $k_{r}<\infty$ we could derive from this the uniform convergence of $p_{\Omega r}$ to a unique limit $p_{r}$ on $V-a$, the capacity function $\gamma$, for which Theorems 25, 26 continue to hold in a class $P$ defined in an obvious manner. If $k_{r}=\infty$, limiting capacity functions still exist but uniqueness is lost. We shall not use limiting functions in either case but introduce:

Definitions. The capacity of the subboundary $r$ of a locally Euclidean space is

$$
c_{\Upsilon}=\frac{1}{k_{\Upsilon}^{2-n}}
$$

A boundary component $\gamma$ is weak if $c_{\gamma}=0$.

We distinguish two classes of locally Euclidean spaces:

$$
\begin{aligned}
& C_{\beta}=\left\{V \mid c_{\beta}=0\right\}, \\
& C_{\gamma}=\{V \mid \text { each boundary component } \gamma \text { is weak }\} .
\end{aligned}
$$

The capacity of a compact set in a solid sphere $|z|<\rho$ of $R^{n}$ can also be defined on replacing the singularity $(58)$ by $|z|^{2-n}\left(\omega_{n}(n-2)\right)^{-1}+h(z)$ in $|z| \geqq \dot{\rho}$. 
with $h(z) \rightarrow 0$ as $|z| \rightarrow \infty$.

\section{§. Classification of locally Euclidean spaces}

We have arrived at four classes of locally Euclidean spaces: $O_{H B}, O_{H D}, C_{B}$, $C_{\Upsilon}$. We conclude our study by introducing other significant classes and by listing problems they lead to.

28. A Green's function $g_{\Omega}(z, a)$ for a regular region $\Omega$ has, by definition, the singularity $|z-a|^{2-n}$ at $a$, and $g \mid \partial \Omega=0$. By the maximum principle $g_{\Omega} \leqq g_{\Omega^{\prime}}$ for $\Omega \subset \Omega^{\prime}$, and the directed limit $g_{V}=\lim _{\Omega \rightarrow 1} g_{\Omega}$ either exists or is $\infty$ in $V$. In the former case it is called the Green's function in $V$. A space $V$ is said to be parabolic, $V \in O_{G}$, if it has no Green's function; otherwise it is hyperbolic. We note in passing:

Every region $V \subset R^{n}$ is hyperbolic.

In fact, every $g_{\Omega}, \Omega \subset V$, is dominated by the Green's function $|z-a|^{2-n}$ of $R^{n}$

29. In strict analogy with the concept of the real part of an analytic function in the 2-dimensional case we introduce:

Definition. The class $R$ for $V$ consists of harmonic functions in $V$ with vanishing fux across every component $\gamma_{\Omega j}$ of the boundary $\beta_{\Omega}$ of every regular region $\Omega$ of $V$ :

$$
\int_{r_{\Omega j}} \frac{\partial u}{\partial n} d S=0
$$

If the span $S$ is defined for the canonical partition, the preceding reasoning for $H D$ applies to $R D$ and we obtain :

$$
O_{R B} \subset O_{R D} \text {. }
$$

30. In a canonical exhaustion each $\beta_{\Omega j}$ has the property that $V-\beta_{\Omega j}$ consists of two components. We shall refer to such hypersurfaces $\beta_{\Omega j}$ as dividing cycles.

Definition. The class $K$ for $V$ is composed of harmonic functions in $V$ with vanishing flux across every dividing cycle.

For $V \subset R^{n}$ the classes $R$ and $K$ coincide. For an $n$-dimensional $V$ imbedded in a higher dimensional $R^{n}$, they differ in general. 
31. In $R^{2 n}=C^{n}$ we consider the class $A$ of analytic functions of $n$ complex variables and the class of real parts of such functions.

32. Let $p_{\Omega}$ be the capacity function of $\partial \Omega=\beta_{\Omega}$ in $\Omega$ with singularity

$$
-s=-r^{2-n} /\left(\omega_{n}(n-2)\right)
$$

(cf. No. 19) at a given point $a \in \Omega$.

Definition. The class $H M_{q}$ in a locally Euclidean space $V$ consists of those $u \in H$ on $V$ for which the mean $(q \geqq 1)$

$$
M=\int_{\beta \Omega}|u|^{q} \frac{\partial p_{\Omega}}{\partial n} d S
$$

is bounded for all $\Omega \subset V$.

33. In analogy with $\log |w|$ of a meromorphic function $w$ on a plane region we introduce: $L$ is the class of harmonic functions in a given locally Euclidean space $V \subset R^{n}$ with singularities $c_{j} s$ at isolated points $z_{j}, j=1, \ldots$, the coefficients $c_{j}$ being nonzero real numbers.

Given $a \in V$ and $v \in L$ on $V$, take a regular region $\Omega$ containing $a$ and decom. pose $v \mid \beta_{\Omega}$ into $v^{+}=\max (v, 0)$ and $v^{-}=\max (-v, 0)$. Let $x_{\Omega}^{+}, x_{\Omega}^{-}$be the solutions in $S$ of the Dirichlet problem with boundary values $v^{+}, v^{-}$, respectively.

Let $a_{\mu}\left(\mu=1, \ldots, \mu_{\Omega}\right), b_{\nu}\left(\nu=1, \ldots, \nu_{\Omega}\right)$ be the positive and negative singularities of $v$ in $\Omega$. Denote by $g_{\Omega}\left(z, z_{j}\right)$ the Green's function on $\bar{\Omega}$ with singularity $s$ at $z_{j}$ and set

$$
\begin{aligned}
& y_{\Omega}^{+}(z)=\sum_{a \mu \in \Omega} g_{\Omega}\left(z, a_{\mu}\right), \quad y_{\Omega}^{-}(z)=\sum_{u_{\nu} \in \Omega} g_{\Omega}\left(z, b_{\nu}\right), \\
& u_{\Omega}^{+}=x_{\Omega}^{+}+y_{\Omega}^{+}, \quad u_{\Omega}^{-}=x_{\Omega}^{-}+y_{\Omega}^{-} .
\end{aligned}
$$

Definitions. The caracteristic $C(\Omega)$ of $v \in L$ is

$$
C(\Omega)=u_{\Omega}^{+}(a) .
$$

The class LC of functions of bounded characteristic in a locally Euclidean space $V$ consists of $v \in L$ with bounded $C(\Omega)$ for all $\Omega \subset V$.

We can thus speak of harmonic functions of bounded characteristic without reference to meromorphic functions.

34. Let $P$ stand for positive. We have introduced classes $I J$ with $I=H$, $K, A, R, L$ and $J=P, B, D, M_{q}, C$. Some of the classes, such as $L B$, are 
obviously void, and we only consider nondegenerate classes.

Given a locally Euclidean space $V$ let $\widetilde{V}_{1}$ be the complement of a regular region with border $\alpha_{1}$. With each nondegenerate class $I J$ we associate the class $I_{0} J$ of functions $u \in I J$ on $\bar{V}_{1}$ with $u \mid \alpha_{1}=0$. Such functions are useful in studying removability properties of the boundary.

35. A general classification theory can be developed for locally Euclidean spaces. As special cases one can consider regions in $R^{n}$, and $n$-dimensional submanifolds in a higher dimensional space $R^{m}$. The following problems arise:

(1) What are the inclusion relations between the various classes $O_{I J}, O_{I_{0} J}$, $C_{\beta}, C_{\curlyvee}$ ? Do the classes $O_{I_{0} J}$ for a fixed $I$ coincide (cf. [5])?

(2) Which inclusion relations are strict for $V^{n} \subset R^{n}$, which for $V^{n} \subset R^{m}$, and which for locally Euclidean spaces $V$ ? Do the classes $O_{I J}$ for a fixed $I$ generally coincide in the first case? Is $O_{H M_{q}}=O_{H P}$ for $q=1$, but $O_{H M_{q}}=O_{H B}$ for $q>1$ (cf. [2]). Can counterexamples be constructed by removing from the unit ball equidistant radial segments of "meridian" planes and by suitably identifying the "faces" of such segments?

(3) The modulus of a regular region $\Omega$ of a locally Euclidean space $V$ can be defined analogously to that on Riemann surfaces. Can $\Omega$ be subdivided into two regular regions each with a modulus arbitrarily close to 1 ? Are there modular tests for a given $V$ to belong to a given class?

(4) Can tests in terms of deep coverings or of Riemannian metrics be formed (cf. [2])?

(5) What metric properties do the boundaries of $V^{n} \subset O_{I J}, C_{\beta}, C_{\curlyvee}$ possess in $R^{n}$ or $R^{m}$ (cf. [1])?

(6) In what classes are the complements $R^{n}-C$ and $R^{n}-S$ of the $n$ dimensional analogues of Cantor sets $C$ and Schottky sets $S$ (cf. [47)? What can be said about their complements with respect to compact locally Euclidean spaces (cf. problem (8))?

(7) Is the complement of a generalized Cantor set in some class $O_{1 J}$ if and only if the volume $\Pi_{1}^{\infty}\left(1-\left(1 / p_{n}\right)\right)^{n}$ vanishes [4]?

(8) Compact locally Euclidean spaces can be formed by identifying opposite faces of an $n$-cube. Can unramified Abelian covering spaces [4] be formed and do they all belong to an $O_{I J}$ ?

(9) Remove a disk $D$ from $R^{n}$ and take two copies $V_{1}, V_{2}$, of the remaining 
space. Identify the upper (lower) face of $D$ in $V_{1}$ with the lower (upper) face of $D$ in $V_{2}$ so as to form a locally Euclidean covering space of $R^{n}$. More generally, construct covering spaces of the "cube" of (8) by removing several disks and using several duplicates of the remaining space, in the same manner as forming covering surfaces of $R^{2}$, with the branch points replaced by circles, the connecting line segments by encircled disks. Develop a classification of such covering spaces based on the ramification properties, in analogy with the classical type problem.

(10) If the potential $p$ of a unit mass distribution $d \mu$ on a compact set $E$ in $R^{n}$ is defined as

$$
p(z)=\int_{E}\left(|z-\zeta|^{2-n} / \omega_{n}(n-2)\right) d \mu(\zeta),
$$

what is the relation between the equilibrium potential and our capacity function $[7,2]$ ?

(11) Is the component $\gamma$ of a compact set $E$ in $R^{n}$ a point if and only if $c_{\Upsilon}=0$ (cf. $[8]$ )?

(12) Can an "equivalence" of locally Euclidean spaces, (or at least of $n$ manifolds $V$ in $R^{m}$ or in $R^{2 m}=C^{m}$ ) be defined in terms of isomorphisms of suitable function spaces or by quasiconformality?

(13) In the affirmative case, is a component $\gamma$ of $\partial V$ always a point or always a continuum or are there "unstable" components [8]?

(14) Cover $R^{n}$ with a set of cubes with side 1 and arrange the cubes in a sequence $\left\{Q_{i}\right\}$ such that the $R_{j}=\cup_{1}^{j} Q_{i}, j=1,2, \ldots$, form a nested sequence of regions exhausting $R^{n}$. For $\varepsilon>0$ remove from $Q_{i}$ a Cantor set $C_{i}$ such that $Q_{i}-C_{i}$ has volume $2^{-i} \varepsilon$. Then the region $V=R^{n}-\cup_{1}^{\infty} C_{i}$ has an arbitrarily small volume $\varepsilon$, yet is dense in $R^{n}$. Does $V$ have an equivalent $V^{*}$ in $R^{n}$ (at least if $n=2 \mathrm{~m}$ ) such that one boundary component of $V^{*}$ is a continuum? Can $V^{*}$ be a bounded region?

(15) Under what self-mappings of $R^{n}$ is a class $O_{1 J}$ preserved? In particular, what can be said about quasi-conformally equivalent regions?

(16) Can the classification theory be extended to mappings of the complex space $C^{n}$ into itself, with suitable modifications of properties $P, B, \dot{D}, M, C$ ?

(17) To what extent can an analogue of the theory of meromorphic functions of bounded characteristic be developed for $L C$ ? In particular, can func- 
tions in $L C$ be decomposed into extremal $L P$-functions? Do the Poisson-Stieltjes formula and the decompositions by Parreau and Rao generalize?

(18) Can a value distribution theory be developed for analytic functions suitably associated with harmonic functions in locally Euclidean spaces?

(19) Can the following interpolation problem be solved in terms of linear combinations of functions $p_{0}-p_{1}$ with suitable singularities: given a locally Euclidean space $V$, points $z_{1}, \ldots, z_{m} \in V$, and real numbers $r_{1}, \ldots, r_{m}$, find a harmonic function $u$ in $V$ with $u\left(z_{i}\right)=r_{i}, i=1, \ldots, m$, and such that the Dirichlet integral is minimized?

\section{REFERENCES}

[1] L. Ahlfors and A. Beurling, Conformal invariants and function-theoretic null-sets, Acta Math., 83 (1950), 101-129.

[2] L. Ahlfors and L. Sario, Riemann surfaces, Princeton University Press, 1960, $382 \mathrm{pp}$.

[3] G. Fichera, Alcuni recenti sviluppi della teoria dei problemi al contorno per le equazioni alle derivate parziali lineari, Convegno internationale sulle equazioni lineari alle derivate parziali, Trieste, 1954, Editioni Cremonese, Roma, 1955, $231 \mathrm{pp}$.

[4] L. Sario, Über Riemannsche Flächen mit hebbarem Rand, Ann. Acad. Sci. Fennicae A. I., 50 (1948), 1-79.

[5] L. Sario, Sur la classification des surfaces de Riemann, C. R. Onzième Congr. Math. Scand. Trondheim, 1949, 229-238.

[6] L. Sario, A linear operator method on arbitrary Riemann surfaces, Trans. Amer. Math. Soc., 72 (1952), 281-295.

[7] L. Sario, Capacity of the boundary and of a boundary component, Ann. of Math., (2) 59 (1954), 135-144.

[8] L. Sario, Strong and weak boundary components, J. Analyse Math., 5 (1956/57), 389398.

University of California, Los Angeles 